\title{
Autonomous Way Detection for Visually Challenged using Machine Learning Algorithm
}

\author{
R. Priyadharshini, J. Ramya, S. Sandhiya, \\ Dept of Electronics and Communication \\ Engineering, Panimalar Engineering College, \\ Chennai-123
}

\begin{abstract}
This paper presents a Smart system for visually impaired, that make use of ultrasonic sensor and camera. The main aim of this work is to design a voice-based alerting system for the blind people. Visually impaired individuals find navigation difficult as they struggle every day in performing actions for bypassing obstacles and hurdles in their path. In order to help blind people, navigate safely and quickly this system is proposed. Ultrasonic sensor is placed on the spectacle which is used for obstacle detection with distance indication. The camera is used to detect the object in front of the blind people and alert them using APR voice module. This system prevents the blind people accidents and identifies the object in front of them.
\end{abstract}

Keywords: Indoor localization, speeded up robust features, $K$ nearest neighbors.

\section{INTRODUCTION}

The visually impaired people usually have difficulties in walking in an unfamiliar and complex place independently. Visually impaired individuals find navigation difficult as they struggle every day in performing actions for by passing obstacles and hurdles in their path. In the proposed system. The voice based alert system for the visually impaired people is implemented. This paper presents a Smart system for visually impaired, that make use of ultrasonic sensor and camera. The main aim of this work is to design a voice-based alerting system for the blind people. The ultrasonic sensor is used to detect the range of the objects, when the objects near to the blind people it sends the alert message to the controller, the camera module and captures the obstacle in front of them. The camera is used to detect the object in front of the blind people and alert them using APR voice module. This system prevents the blind people accidents and identifies the object in front of them. The device has to know where the person is located in order to make a correct decision for guiding the person. This refers to be the indoor localization problem. The GPS and GSM help the blind people during the outdoor navigation. This method prevents the blind people accidents and blind people know the obstacle type.

\section{RELATED WORKS}

An affordable solution to help blind people navigate unknown environments is presented [1]. It performs image classification on a Raspberry $\mathrm{Pi}$ and provides feedback to users by means of vibration motors to signal the presence of an obstacle in a given direction. The training phase is performed off-line, while the on-line phase can classify an

\author{
Mrs. Jeneetha Jebanazer M.E., \\ Associate Professor, \\ Dept of Electronics and Communication \\ Engineering \\ Panimalar Engineering College, Chennai-123
}

image in 1.12 seconds on average. Provides an evaluation using several thousand images, showing that a precision of $79 \%$ and a recall of $79 \%$ is achieved. An overview of both early and recent developments in artificial aids for blind orientation and mobility is presented [2]. Most aids developed previously fall into the category of "mobility aids" intended to sense obstacles in or near the traveler's immediate path. The more global problem of absolute orientation and navigation has not been well addressed by researchers in electronic aids, but this situation has changed. A new system for aiding blind orientation, described here, is now advancing beyond the experimental stage. The last decades a variety of portable or wearable navigation systems have been developed to assist visually impaired people during navigation in known or unknown, indoor or outdoor environments. There are three main categories of these systems: Electronic travel aids (ETAs), electronic orientation aids (EOAs), and position locator devices (PLDs). A comparative survey among portable/wearable obstacle detection/ avoidance systems (a subcategory of ETAs) in an effort to inform the research community and users about the capabilities of these systems and about the progress in assistive technology for visually impaired people. [3] is based on various features and performance parameters of the systems that classify them in categories, giving qualitative-quantitative measures. Finally, it offered a ranking, which will serve only as a reference point and not as a critique on these systems.

A new model of a mobility aid for the blind was designed using microprocessor and ultrasonic devices. Evaluation based on psychophysical experiments. [4], a down swept FM ultrasound signal is emitted from a transmitting array with broad directional characteristics in order to detect obstacles. The ultrasound reflections from the obstacles are picked up by a two-channel receiver. The frequency of the emitted ultrasound is swept from 70 to $40 \mathrm{kHz}$ within $1 \mathrm{~ms}$, so it has almost the same characteristics as the ultrasound a bat produces for echolocation. The frequency of the reflected ultrasound wave is down converted by about 50: 1 by using a microcomputer with $\mathrm{A} / \mathrm{D}$ and $\mathrm{D} / \mathrm{A}$ converters. These audible waves are then presented binaurally through earphones. In this method obstacles may be perceived as localized sound images corresponding to the direction and the size of the obstacles. From the results of psychophysical experiments, it was found that down swept FM ultrasound was superior for the recognition of small obstacles compared to other ultrasonic schemes. With it a blind person can 
recognize a 1-mm-diameter wire. It was also proved that the blind could discriminate between several obstacles at the same time without any virtual images. [4] modeled after the bat's echolocation system, is very effective at detecting small obstacles placed in front of the head.

Obstacle recognition system for the blind people using the Radio frequency identification (RFID) is presented in [5]. In order to walk without being accompanied, people with visual impairment use touching clues such as canes. The main concern of blind people is to be able to detect any type of obstacle within their surroundings. For that, several techniques have been developed such as Differential Global Positioning System (DGPS) or Radio-frequency identification (RFID). [5] aims on developing a blind recognition system through canes using RFID and covers a brief insight of relevant work about the RFID system. The review of RFID system introduces the technical features of this utility and presents the concept of research being developed in smart canes for blind people.

The increasing use of digital technology, availability of economical image capturing devices like mobile phones, digital cameras etc, need of powerful technology to aid blind or visually impaired people, attracting researchers to the problem of recognizing text in images. Detecting text from scene image is more difficult as compared to that from printed documents. Lots of research has been done on detecting scene text to overcome certain challenges like perspective distortion, aspect ratio, font size, etc. Speed, complexity, cost and accuracy are important parameters must be taken into consideration while designing such systems. Computer vision is one of the emerging technologies that can be used to aid visually impaired people for navigation (both indoor and outdoor), accessing printed material, etc. [6] describes an approach to extract and recognize text from scene images effectively using computer vision technology and to convert recognized text into speech so that it can be incorporated with hardware to develop Electronic travel aid for visually impaired people in future.[7] presents a new concept for a travel aid for the blind. A prototype device, called the NavBelt, was developed to test this concept. The device can be used as a primary or secondary aid, and consists of a portable computer, ultrasonic sensors, and stereophonic headphones. The computer applies navigation and obstacle avoidance technologies that were developed originally for mobile robots. The computer then uses a stereophonic imaging technique to process the signals from the ultrasonic sensors and relays their information to the user via stereophonic headphones.

The user can interpret the information as an acoustic "picture" of the surroundings, or, depending on the operational mode, as the recommended travel direction. The acoustic signals are transmitted as discrete beeps or continuous sounds. Experimental results with the NavBelt simulator and a portable prototype show that users can travel safely in an unfamiliar and cluttered environment at speeds of up to $0.8 \mathrm{~m} / \mathrm{s}$.

\section{PROPOSED SYSTEM}

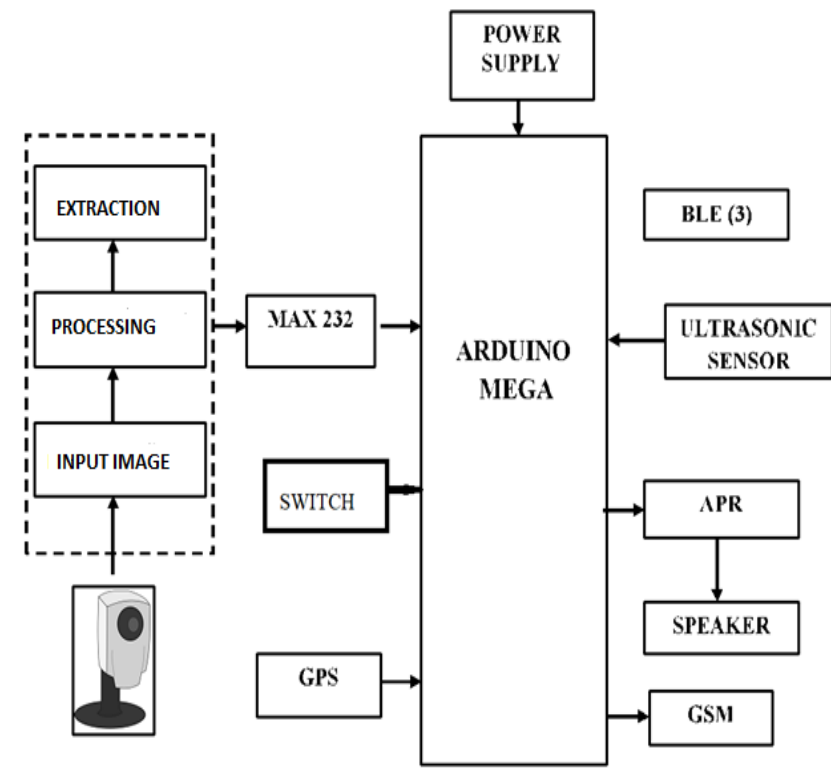

In the proposed system, we have implemented the voice based alert system for the visually impaired people. The ultrasonic sensor is used to detect the range of the objects, when the objects near to the blind people it sends the alert message to the controller. The camera module captures the obstacle in front of them and matches the images with the predefined images in MATLAB and it tells the blind person about the type of object via APR module and speaker. The Bluetooth in their smart phone guides the blind people when they reach the location.

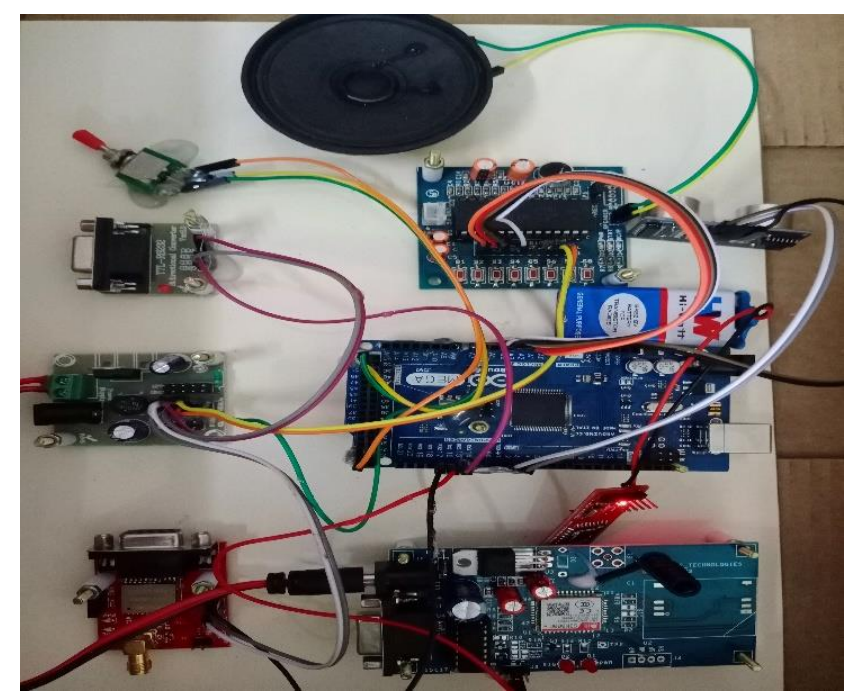

The BLE (BLUETOOTH LOW ENERGY) module activates and informs them through smart phone speaker. The GPS and GSM help the blind people during the outdoor navigation. This method prevents the blind people accidents and blind people know the obstacle type.

\section{ARDUINO MEGA}

The MEGA 2560 is designed for more complex projects. With 54 digital I/O pins, 16 analog inputs and a larger space for your sketch it is the recommended board for 
3D printers and robotics projects. This gives your projects plenty of room and opportunities. The Arduino Mega 2560 is a microcontroller board based on the ATmega2560. It has 54 digital input/output pins (of which 15 can be used as PWM outputs), 16 analog inputs, 4 UARTs (hardware serial ports), a $16 \mathrm{MHz}$ crystal oscillator, USB connection, a power jack, an ICSP header, and a reset button.

\section{ULTRASONIC SENSOR}

An ultrasonic sensor is an instrument that measures the distance to an object using ultrasonic sound waves to produce distinct echo patterns. When an electrical pulse of high voltage is applied to the ultrasonic transducer it vibrates across a specific spectrum of frequencies and generates a burst of sound waves. Whenever any obstacle comes ahead of the ultrasonic sensor the sound waves will reflect back in the form of echo and generates an electric pulse. It calculates the time taken between sending sound waves and receiving echo. The echo patterns will be compared with the patterns of sound waves to determine detected signal's condition.

\section{GLOBAL POSITIONING SYSTEM}

GPS or Global Positioning System is a satellite navigation system that furnishes location and time information in all climate conditions to the user. GPS is used for navigation in planes, ships, cars and trucks also. The system gives critical abilities to military and civilian users around the globe. GPS provides continuous real time, 3dimensional positioning, navigation and timing worldwide. The Global Positioning System (GPS) is a satellite-based navigation system made up of at least 24 satellites.

\section{GLOBAL SYSTEM FOR MOBILE COMMUNICATION}

Global system for mobile communication (GSM) is a globally accepted standard for digital cellular communication. The GSM standard is intended to address this problem. GSM/GPRS Modem-RS232 is built with Dual Band GSM/GPRS engine- SIM900, works on frequencies 900/ $1800 \mathrm{MHz}$. The Modem is coming with RS232 interface, which allows you connect PC as well as microcontroller with RS232 Chip(MAX232). The baud rate is configurable from 9600-115200 through AT command.

\section{APR 9600 VOICE IC}

APR9600 is a low-cost high-performance sound record/replay IC incorporating flash analogue storage technique. Recorded sound is retained even after power supply is removed from the module. The replayed sound exhibits high quality with a low noise level. Sampling rate for a 60 second recording period is $4.2 \mathrm{kHz}$ that gives a sound record/replay bandwidth of $20 \mathrm{~Hz}$ to $2.1 \mathrm{kHz}$. However, by changing an oscillation resistor, a sampling rate as high as $8.0 \mathrm{kHz}$ can be achieved. This shortens the total length of sound recording to 32 seconds.

\section{BLUETOOTH LOW ENERGY}

Bluetooth Low Energy (BLE) possess the central role and provides APIs that apps can use to discover devices, query for services, and transmit information. Transferring small amounts of data between nearby devices. Interacting with proximity sensors like Google Beacons to give users a customized experience based on their current location. In contrast to Classic Bluetooth, Bluetooth Low Energy (BLE) is designed to provide significantly lower power consumption. This allows Android apps to communicate with BLE devices that have stricter power requirements, such as proximity sensors, heart rate monitors, and fitness devices.

\section{IMAGE PROCESSING}

The term digital image refers to processing of a two-dimensional picture by a digital computer. In a broader context, it implies digital processing of any two-dimensional data. A digital image is an array of real or complex numbers represented by a finite number of bits. An image given in the form of a transparency, slide, photograph or an X-ray is first digitized and stored as a matrix of binary digits in computer memory. This digitized image can then be processed and/or displayed on a high-resolution television monitor.

\section{MATLAB}

\begin{abstract}
MATLAB (matrix laboratory) is a multiparadigm numerical computing environment and proprietary programming language developed by MathWorks which allows matrix manipulations plotting of functions and data, implementation of algorithms, creation of user interfaces, and interfacing with programs written in other languages.
\end{abstract}

\section{EMBEDDED C}

Embedded $\mathrm{C}$ is most popular programming language in software field for developing electronic gadgets. Each processor used in electronic system is associated with embedded software. Embedded $\mathrm{C}$ programming plays a key role in performing specific function by the processor. In day-to-day life we used many electronic devices such as mobile phone, washing machine, digital camera, etc. These all device working is based on microcontroller that are programmed by embedded C.

\section{EMBEDDED SYSTEMS}

Embedded System is a system composed of hardware, application software and real time operating system. It can be small independent system or large combinational system. Embedded System is a way of working, organizing or performing one or many tasks according to a fixed set of rules, program or plan. It is an arrangement in which all the unit combined to perform a work together by following certain set of rules in real time computation. 


\section{RESULTS AND DISCUSSIONS}

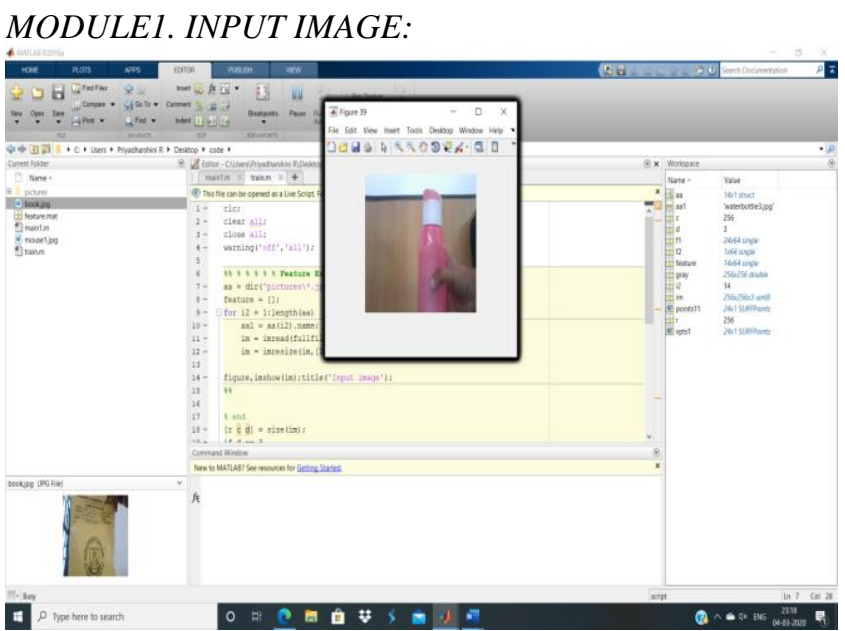

The above figure indicates the captured image of a bottle where we call special MATLAB functions to read and write image data from graphics file formats: To read a graphics file format image use imread.

\section{MODULE2. IMAGEPREPROCESSING}

Preprocessing techniques are needed on color, grey-level or binary document images containing text and/or graphics. In character recognition systems most of the applications use grey or binary images since processing color images is computationally high. Such images may also contain non-uniform background and/or watermarks making it difficult to extract the document text from the image without performing some kind of preprocessing, therefore; the desired result from preprocessing is a binary image containing text only.

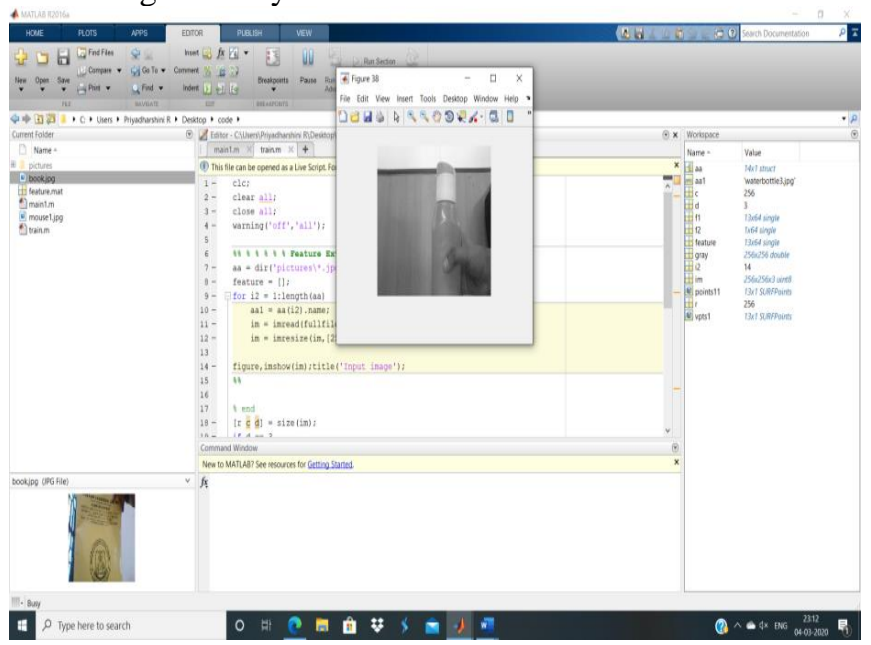

To achieve this, first some image enhancement techniques are used to remove noise or correct the contrast in the image, second, thresholding to remove the background containing any scenes, watermarks and/or noise, third, page segmentation to separate graphics from text, fourth, character segmentation to separate characters from each other and, finally, morphological processing to enhance the characters in cases where thresholding and/or other preprocessing techniques eroded parts of the characters or added pixels to them.

\section{MODULE3: FEATURE EXTRACTION}

Feature extraction is a process of dimensionality reduction by which an initial set of raw data is reduced to more manageable groups for processing.

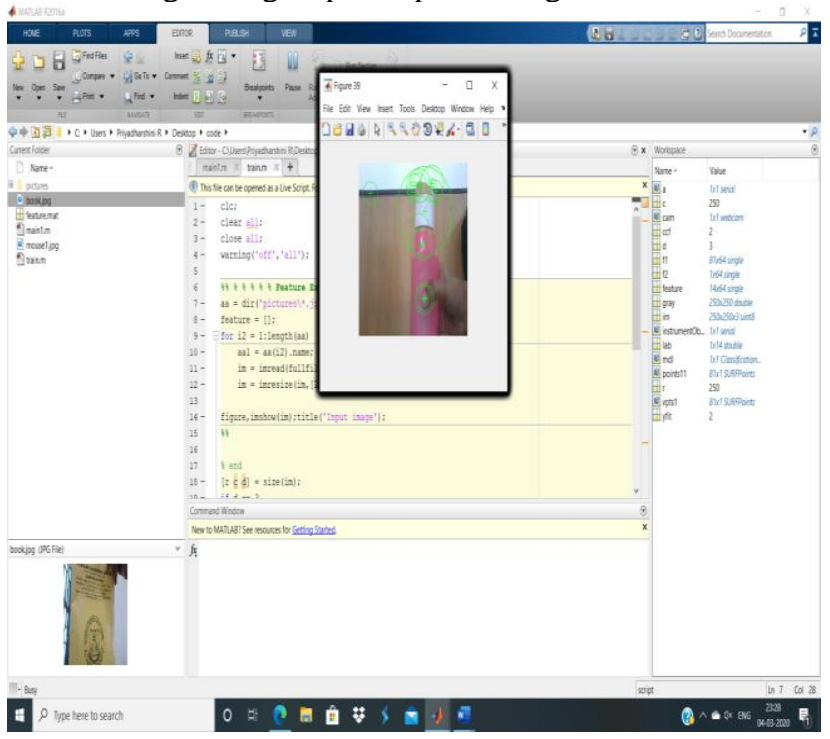

\section{SURF- SPEEDED UP ROBUST FEATURES}

In computer vision, speeded up robust features (SURF) is a patented local feature detector and descriptor. It can be used for tasks such as object recognition, image registration, classification, or 3D reconstruction. It is partly inspired by the scale-invariant feature transform (SIFT) descriptor MODULE4: CLASSIFICATION

Image classification refers to a process in computer vision that can classify an image according to its visual content. For example, an image classification algorithm may be designed to tell if an image contains a human figure or not.

\section{KNN CLASSIFICATION}

The k-nearest neighbors $(\mathrm{KNN})$ algorithm is a simple, supervised machine learning algorithm that can be used to solve both classification and regression problems. It's easy to implement and understand, but has a major drawback of becoming significantly slows as the size of that data in use grows.

\section{CONCLUSION AND FUTURE SCOPE}

In this paper, we have designed an assistive device for visually impaired persons which has the following provisions: 1. Indoor localisation 2 . emergency message about location through GSM 3. Object detection and recognition.

The indoor localisation is implemented using the Bluetooth low energy module. Objects can be detected using ultrasonic sensor. MAT lab algorithms such as SURF and Knn are used for object recognition. The trained images of the objects in the MAT lab is recognised and the type of object is heard as voice through speaker. Thus, this tool will be helpful for visually impaired persons for safe localisation and object recognition. 
For future modification, the indoor localization can be executed using Wi-Fi which can cover larger distance. In our project only, the live location of the person can be shared through GSM. Further it can be developed to provide the facility of live tracking of the person.

\section{REFERENCE}

[1] Dalal Khalid Aljasem, Michael Heeney, Armando PesentiGritti, Franco Raimondi "On- the-fly Image Classification to Help Blind People",2016 12th International Conference on Intelligent Environments.

[2] "New Developments in Mobility and Orientation Aids for the Blind", John a. brabyn, Member, 1982, IEEE

[3] Dimitrios Dakopoulos and Nikolaos G. Bourbakis, Fellow, IEEE, "Wearable Obstacle Avoidance Electronic Travel Aids for Blind:
A Survey", IEEE transcations on systems,man and cyberneticsPart C: Applications and reviews, vol.40, no.1, January 2010.

[4] "A Blind Mobility Aid Modeled After Echolocation of Bats", TohruIfukube, Tadayuki Sasaki, and Chen Peng, 1991, IEEE.

[5] M. Nassih, I. Cherradi, Y. Maghous, B. Ouriaghli and Y. SalihAlj "Obstacles Recognition System for the Blind People Using RFID",2012 Sixth International Conference on Next Generation Mobile Applications, Services and Technologies.

[6] Akhilesh A. Panchal, Shrugal Varde, M.S. Panse, "Character Detection and Recognition System for Visually Impaired People", IEEE International Conference on Recent Trends in Electronics Information Communication Technology, May 2021, 2016, India.

[7] Shraga Shoval, Johann Borenstein,* Member, IEEE, and Yoram Koren, Senior Member, IEEE, "The Navbelt-A Computerized Travel Aid for the Blind Based on Mobile Robotics Technology" , IEEE transactions on biomedical engineering, vol.45, no.11, November 1998. 